1998

\title{
Noise Sustained Waves In Subexcitable Media: From Chemical Waves To Brain Waves
}

P. Jung

A. Cornell-Bell

F. Moss

S. Kadar

J.Wang

See next page for additional authors

Follow this and additional works at: https://researchrepository.wvu.edu/faculty_publications

\section{Digital Commons Citation}

Jung, P.; Cornell-Bell, A.; Moss, F.; Kadar, S.; Wang, J.; and Showalter, K., "Noise Sustained Waves In Subexcitable Media: From Chemical Waves To Brain Waves" (1998). Faculty Scholarship. 615.

https://researchrepository.wvu.edu/faculty_publications/615 
Authors

P. Jung, A. Cornell-Bell, F. Moss, S. Kadar, J. Wang, and K. Showalter 


\title{
Noise sustained waves in subexcitable media: From chemical waves to brain waves
}

\author{
P. Jung ${ }^{a)}$ \\ Department of Physics and Astronomy, Program for Condensed Matter and Surface Science, \\ and Program for Neurobiology, Ohio University, Athens, Ohio 45701
}

\author{
A. Cornell-Bell \\ Viatech Imaging, Ivoryton, Connecticut 06442 \\ F. Moss \\ Department of Physics and Center for Neuroscience, University of Missouri, St. Louis, Missouri 63121 \\ S. Kadar, J. Wang, and K. Showalter \\ Department of Chemistry, West Virginia University, Morgantown, West Virginia 26506
}

(Received 18 February 1998; accepted for publication 4 May 1998)

\begin{abstract}
We discuss a novel type of spatiotemporal pattern that can be observed in subexcitable media when coupled to a thermal environment. These patterns have been recently observed in several different types of systems: a subexcitable photosensitive Belousov-Zhabotinsky reaction, hippocampal slices of rat brains, and astrocyte syncytium. In this paper, we introduce the basic concepts of subexcitable media, describe recent experimental observations in chemistry and neurophysiology, and put these observation into context with computer simulations. (c) 1998 American Institute of Physics.
\end{abstract}

[S1054-1500(98)01203-8]

Pattern formation in spatially extended dynamic systems is often described in terms of nonlinear partial differential equations for macroscopic variables. Fluctuations, e.g., due to a finite number of molecules in chemical reactions, can usually be neglected. Here we discuss fluctuations stemming from the coupling of the extended dynamic system to a fluctuating environment. They do not scale with the size of the system and are therefore not negligible. We show that these fluctuations become important close to the threshold of pattern formation, where they indeed dominate the spatiotemporal behavior of the system. Below, but close to the threshold, fluctuations can maintain and control the geometric features of large-scale patterns. This is demonstrated numerically and experimentally with a photosensitive BelousovZhabotinsky reaction. Well below the threshold of pattern formation, noise can sustain locally coherent patterns. The patterns exhibit a spatiotemporal fragmentation statistics that is described by a power law. We report evidence of such a behavior for calcium wave networks in interconnected brain cells.

\section{INTRODUCTION}

Since the early days of nonequilibrium statistical physics, noise has been recognized to play an important role in the formation of ordered states far from thermal equilibrium.

Stochastic resonance ${ }^{1,2}$ represents an important milestone in the theory of noise-induced phenomena. Here, the noise enhances the response of a system to a weak time-

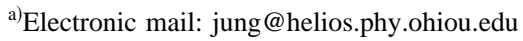

dependent external force by, e.g., synchronizing barrier crossings between metastable states with the external force. The archetype model for studies of SR is a Brownian particle in a bistable potential driven by a homogeneous periodic force (see, e.g., the reviews ${ }^{3,4}$ ). At a certain optimal noise level, the noise-induced escape becomes synchronized with the periodic modulation of the barrier height. The hopping of the particle then exhibits a significant periodic component, quantified by a variety of features of the power spectrum. SR has been discussed also for coupled bistable systems that are each driven by an identical periodic force (Refs. 5-10). The coupling can facilitate a significant enhancement of the periodicity of the response of a single bistable element to the periodic forcing, hence the term array enhanced stochastic resonance. This effect has been observed in a chain of coupled diode resonators by Löcher and Hunt. ${ }^{11}$

The conceptually different phenomenon of spatiotemporal stochastic resonance $e^{12,13}$ has been observed first in an excitable medium below the threshold of pattern formation. The medium is driven by a weak spatiotemporal forcing such as a wave train or a single wave front and the spatiotemporal response is enhanced in the presence of an optimal dose of noise. Spatiotemporal stochastic resonance has been studied theoretically since then in a variety of models. ${ }^{14}$ Experimental evidence has been recently reported in a photosensitive Belousov Zhabotinsky (BZ) reaction ${ }^{15}$ and in a chain of unidirectionally coupled diode resonators. ${ }^{16}$

In Sec. II of this paper, we briefly review excitable dynamics by using the Fitzhugh-Nagumo equations as a working model for neuronal discharge dynamics. We then introduce a dynamical model for the photosensitive Belousov Zhabotinsky reaction, where the threshold of excitation can be controlled by illumination. We then discuss a three-state 
model for excitable dynamics and the role of fluctuations.

In Sec. III, we introduce the concept of subexcitable media, i.e., media that are excitable but not capable of maintaining spatiotemporal patterns such as rotating spiral waves. We discuss a model for the subexcitable photosensitive Belousov-Zhabotinsky reaction that is based on a system of coupled reaction-diffusion equations and a cellular model that is based on a three-state model.

In Sec. IV, we discuss in detail the spatiotemporal patterns of a subexcitable medium that is close but below the threshold of pattern formation, obtained from computer simulations of the cellular model introduced in Sec. III. The global geometric features of these patterns can be controlled by the noise level. We then show the corresponding experimentally observed patterns in the subexcitable photosensitive Belousov-Zhabotinsky reaction under similar slightly subthreshold conditions.

In Sec. V, we consider the case where the medium is well below the threshold of pattern formation. Here noise can still sustain patterns, but these patterns, although coherent on a small scale, are disordered on a large scale (thermal patterns). Using the technique of coherent cluster analysis, we then demonstrate that the thermal patterns exhibit some features of avalanches discussed in models that show selforganized criticality such as sandpile models.

In Sec. VI, we relate the thermal patterns, discussed in Sec. V, to calcium waves in cultured networks of rathippocampal astrocytes and hippocampal slices of rat brains. Those calcium waves share a number of features with the thermal patterns, including their quantitative statistical properties (obtained from the analysis of coherent clusters). There is experimental evidence that the cell-culture operates in a self-organized critical regime.

\section{EXCITABLE DYNAMICS}

Excitability is an important paradigm for a quantitative description of many systems in nature. An excitable system is characterized by three states: the quiescent state, the excited state, and the recovery state. In this section, we discuss zero-dimensional excitable systems, i.e., we neglect spatial gradients of all observables. An example of an excitable system is the Fitzhugh-Nagumo model ${ }^{17}$ for firing of neurons, given by the two-dimensional dynamical system,

$$
\begin{aligned}
& \epsilon \dot{v}=v(a-v)(v-1)-w, \\
& \dot{w}=v-d w-b .
\end{aligned}
$$

The variable $v$ describes the membrane voltage, while $w$ describes the slow $(\epsilon \rightarrow 0)$ recovery process. The nullclines, i.e., the curves where $\dot{v}$ and $\dot{w}$ vanish, are shown in Fig. 1 for $b=0.0, a=0.5$, and $d=1$. The stationary states are given by the intersection of the nullclines. The two nullclines intersect once at $v=0, w=0(S)$, where the intersection point represents a stable node. A small perturbation, which does not bring the system beyond $P$, leads to a relaxation back toward the stationary state $S$. If a perturbation is large (beyond $P$ ), the system switches to the excited state $T_{1}$ (it fires) and returns to the stationary state $S$ only after a large excursion

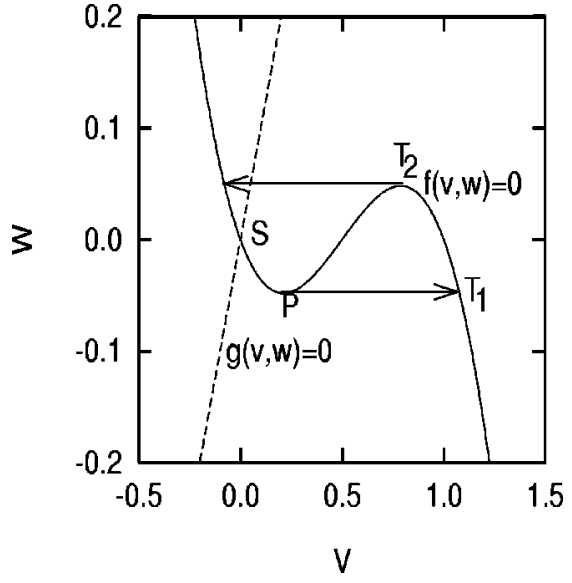

FIG. 1. The nullclines of Eq. (1) are shown for $b=0, a=0.5$, and $d=1$. The intersection $(S)$ of the nullclines at $v=0, w=0$ represents a stable node. The arrows indicate the course of a large excursion if the threshold of excitation $(P)$ is crossed.

$\left(P \rightarrow T_{1} \rightarrow T_{2} \rightarrow S\right)$. During the time interval when the system approaches the stationary state, it is in the recovery state.

Another prominent example of excitable dynamics is the $\mathrm{BZ}$ reaction. In dimensionless units, the reaction is described by the three coupled differential equations for the dimensionless concentration $u$ of $\mathrm{HBrO}_{2}, w$ of $\mathrm{Br}^{-}$and $v$ of the catalyst: $^{18,19}$

$$
\begin{aligned}
& \dot{u}=\frac{1}{\epsilon}\left(q w-u w+u-u^{2}\right), \\
& \dot{w}=\frac{1}{\delta}(\phi-q w-u w+f v), \\
& \dot{v}=u-v .
\end{aligned}
$$

Equations (2) describe the time evolution of the homogeneous concentrations of the relevant chemical species in the photosensitive BZ reaction under illumination. The parameter $\phi$ is added to account for the bromide production from irradiation. ${ }^{20,21}$ The light intensity controls the photochemical production of $\mathrm{Br}^{-}$, an inhibitor of autocatalysis in the $\mathrm{BZ}$ reaction. The parameters $q, \epsilon$, and $\delta$ describe the time scales of the three variables. The variable $w$ is the fastest variable, and can be eliminated adiabatically, yielding

$$
\begin{aligned}
& \dot{u}=\frac{1}{\epsilon}\left(\frac{q-u}{q+u}(f v+\phi)+u-u^{2}\right), \\
& \dot{v}=u-v .
\end{aligned}
$$

Insights into the dynamical properties of the system of differential equations (3) can be obtained by plotting the nullclines $\dot{u}=0$ and $\dot{v}=0$. The nullclines are given by

$$
\begin{aligned}
& v(u)=u, \\
& v(u)=\frac{1}{f}\left(\frac{q+u}{q-u}\left(u^{2}-u\right)-\phi\right) .
\end{aligned}
$$

The nullclines in Eq. (4) are plotted in Fig. 2 for $f=1$ and various values of $\phi$. The nullcline $\dot{u}=0$ has an S-type shape. The branch with the positive slope is unstable. For 


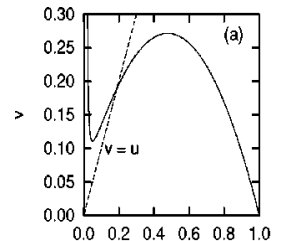

$\mathrm{u}$

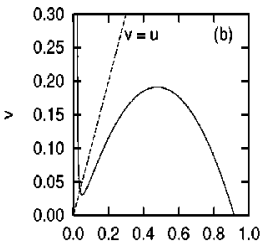

u

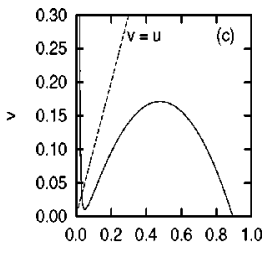

$u$
FIG. 2. The nullclines of Eq. (4) are shown at $\phi=0$ (a), $\phi=0.08$ (b), and $\phi=0.10$ (c) at $f=1$ and $q=0.02$. The intersection of the nullclines in (a) represents an unstable fixed point indicating an oscillatory BZ reaction. In (b) and (c), the intersection represents a stable fixed point and therefore an excitable system.

$0<\phi<4 q$, the two nullclines intersect in this unstable regime, and the BZ reaction is oscillatory. For $\phi>4 q$, the intersection is on a stable branch of the nullcline $\dot{u}=0$ and represents a stable fixed point. In this regime, the system is excitable. Small perturbations (e.g., noise) to $\phi$ shift the nullcline $\dot{u}=0$ up and down generating at occasions an unstable fixed point and thus the onset of the oscillatory reaction. Operating the $\mathrm{BZ}$ reaction at a relatively large value of $\phi$, the excitability is small (i.e., the threshold for excitation is large).

Based on the qualitative features of excitable systems, one can construct a simplified version of excitable dynamics modeled by a three-state system. The three states are the quiescent state $Q$ (corresponding to the stable fixed point $S$ ), the excited state $E$ (corresponding in Fig. 1 to the escape from $P$ to $T_{1}$, and the recovery state $R$ (corresponding to the state of slowly moving back to $S$ ). The state of the system is determined by an input variable $v(t)$. Whenever the variable $v(t)$ crosses a threshold (corresponding to $P$ in Fig. 1), the system switches from the quiescent to the excited state. After some recovery time, the system eventually returns back into the quiescent state. Such a simplified version of excitable dynamics becomes useful for modeling excitable media, since it greatly reduces computational demands in comparison to media based on the continuous models above.

Finally, in this section we discuss the role of noise in excitable systems. In the presence of zero mean Gaussian noise $\xi(t)$, the Fitzhugh-Nagumo model can be written as

$$
\begin{aligned}
& \epsilon \dot{v}=v(a-v)(v-1)-w+\xi(t), \\
& \dot{w}=v-d w-b .
\end{aligned}
$$

Most of the time the noise perturbs the system so weakly that it fluctuates close to the stable fixed point $S$. Occasionally, however, the excitability threshold is crossed and the system runs through a noise-induced large excursion. The rates at which the threshold is crossed can be obtained by applying standard methods in rate theory (for a review of reaction rate theory, see Ref. 22). These spontaneous events have been observed in studies of neurons, emphasizing the role of fluctuations for realistic neural modeling. Stochastic resonance in the noise-driven Fitzhugh-Nagumo model and in other zero-dimensional excitable systems, in general, has been studied by Longtin, ${ }^{23}$ Wiesenfeld et al., ${ }^{24}$ and Collins and collaborators. $^{25}$ The external noise has been shown to enhance periodic and aperiodic signals (aperiodic stochastic resonance $^{26}$ ) in a resonant fashion.

\section{SUBEXCITABLE MEDIA}

In this section we consider spatially extended excitable systems, also known as excitable media. At each point in space, the system is excitable as described in the previous section. Due to the spatial coupling, excitation does not remain localized, but can spread through the medium, giving rise to spatiotemporal patterns of excitation. The generic excitation patterns are target waves (single circular wave fronts) and rotating spiral waves (see, e.g., Ref. 27).

Often, excitable media are described by reactiondiffusion equations. In this paper, we consider twodimensional media with the spatial directions $x$ and $y$. In the case of the Fitzhugh-Nagumo model (1) the membrane potential $v$ and the inhibitor variable $w$ are considered as twodimensional scalar fields $v(x, y, t)$ and $w(x, y, t)$. Locally they obey Eq. (1), but there is also diffusional spread:

$$
\begin{aligned}
& \frac{\partial v}{\partial t}=v(a-v)(v-1)-w+D_{v} \nabla^{2} v, \\
& \frac{\partial w}{\partial t}=v-d w-b+D_{w} \nabla^{2} w .
\end{aligned}
$$

These equations are physiologically only meaningful along one space dimension, describing the propagation of action potentials along axons of neurons. In case of the $\mathrm{BZ}$ reaction and its model, Eqs. (2), various chemicals develop concentration gradients and become scalar fields when the reaction is not stirred. Here, the following reaction diffusion equations apply: $:^{15}$

$$
\begin{aligned}
& \frac{\partial u}{\partial t}=\frac{1}{\epsilon}\left(q w-u w+u-u^{2}\right)+D_{u} \nabla^{2} u, \\
& \frac{\partial w}{\partial t}=\frac{1}{\delta}(\phi-q w-u w+f v)+D_{w} \nabla^{2} w, \\
& \frac{\partial v}{\partial t}=u-v .
\end{aligned}
$$

Noise enters the system through the intensity of the incident light on the reaction cell. ${ }^{15,21}$ The light intensity controls the excitability threshold via the photochemical production of the inhibitor $\mathrm{Br}^{-}$. This allows one to tune the medium into the subthreshold regime, i.e., a regime where the threshold is sufficiently large that a chemical wave cannot be maintained. This is the subexcitable regime.

A discrete version of an excitable medium can be modeled by using the three-state model mentioned in the previous section. ${ }^{12,13}$ We consider a square array of excitable three-state elements $e_{i j}$ at the positions $x_{i j}=i a \hat{x}+j a \hat{y}$ with the unit vectors $\hat{x}$ and $\hat{y}$ in $x$ and $y$ directions, respectively, and the lattice spacing $a$. The firing threshold for each element is denoted by $b$, and the input quantity that controls the state of the element is denoted by $v_{i j}$. The variables $v_{i j}$ are each thermally coupled to a heat bath and therefore undergo fluctuations, described by the Langevin equations, 


$$
\dot{v}_{i j}=-\gamma v_{i j}+\sqrt{\gamma} \xi_{i j}(t)
$$

with Gaussian white noise,

$$
\begin{aligned}
& \left\langle\xi_{i j}(t) \xi_{k l}\left(t^{\prime}\right)\right\rangle=2 \sigma^{2} \delta\left(t-t^{\prime}\right) \delta_{(i j),(k l)}, \\
& \left\langle\xi_{i j}(t)\right\rangle=0 .
\end{aligned}
$$

The constant $\gamma$ describes the rate at which fluctuations decay and $\sigma^{2}$ denotes the spatially homogeneous variance of the fluctuations. Integration of (8) over the finite time step $\Delta t$ yields the map

$$
x_{i j}(t+\Delta t)=x_{i j}(t) \exp (-\gamma \Delta t)+G_{i j},
$$

where $G_{i j}$ are Gaussian distributed random numbers with variance $\sigma^{2}[1-\exp (-2 \gamma \Delta t)]$. When the elements $(k l)$ fire, the emitted $\delta$ spikes are added to the input of the elements ( $i j)$ with weights depending on the spatial distance $r_{(i j)(k l)}$ between the elements $(k l)$ and $(i j)$. Integration over time yields the total contribution of the firing elements $(k l)$ to the input of element $(i j)$ :

$$
\Delta x_{i j}(t+\Delta t)=K \sum_{k l} \exp \left(-\lambda \frac{r_{(i j),(k l)}^{2}}{a^{2}}\right),
$$

where $\lambda$ describes the interaction range and $K$ a coupling constant. Each element undergoes a recovery period after firing. The proper scaling of this model is given by $x_{i j}$ $\rightarrow x_{i j} / b, t \rightarrow \gamma t, \sigma^{2} \rightarrow \sigma^{2} / b^{2}, \gamma \rightarrow \gamma \Delta t, K \rightarrow K / b$. The value of the threshold is normalized to unity.

It has been demonstrated ${ }^{12}$ that this model shows for large coupling $K$ (in the absence of noise) the typical excitation patterns of excitable media, i.e., rotating spiral waves or target waves, usually described in terms of reactiondiffusion equations with two species. ${ }^{27}$ In the presence of noise, the typical excitation patterns can still be observed, but they exhibit rough wave fronts and-depending on the noise level-more serious imperfections such as the breakup of wave fronts and collisions with noise-nucleated waves. The overall picture in the large coupling regime is the coexistence of multiple finite-size cells with coherent patterns.

For weak coupling $K$, however, different phenomena can be observed. To maintain a firing pattern, the coupling $K$ has to exceed a critical value $K_{0}$ which is approximated for small $\lambda$ by ${ }^{28}$

$$
K_{0}(\gamma) \approx \sqrt{\frac{\lambda}{\pi}} \frac{1}{\exp (-\lambda)+\exp (\gamma) \sum_{n=2}^{\infty} \exp \left(-\lambda n^{2}-n \gamma\right)} .
$$

In the subexcitable regime, where $K<K_{0}$, waves cannot be maintained in the absence of fluctuations.

\section{NOISE SUSTAINED SPATIOTEMPORAL PATTERNS IN SUBEXCITABLE MEDIA}

As discussed in the previous section, spatiotemporal patterns cannot persist in subexcitable media in the absence of fluctuations. In the presence of a small amount of noise, however, the excitation threshold will be surmounted along sufficient fractions of the wave front to allow an excitation wave to propagate. The randomness in the selection of ele-

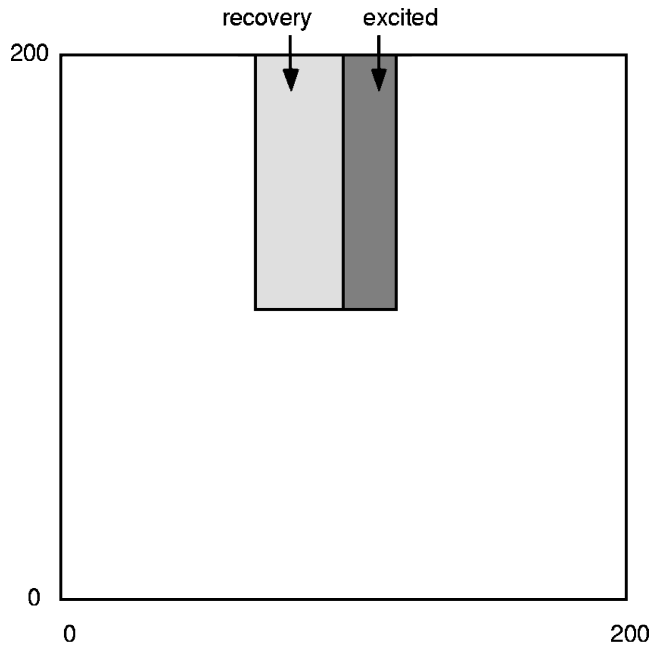

FIG. 3. The square denotes a $201^{*} 201$ array of excitable three-state elements. The initial preparation is a strip of excited elements, and attached to the left a strip of elements in the recovery state. The strip of elements in the recovery state is placed to avoid the propagation of excitation to the left. Additional propagation to the left would interfere with the rotating spiral wave that develops from propagation to the right. In systems with noise, this artificial symmetry breaking is not necessary since noise-induced breakup of wave fronts creates open ends that subsequently curl up to rotating spiral waves in the same way as the initial preparation above.

ments that are carrying the wave creates a certain roughness in the wave front. When the variance of the noise becomes larger, more pronounced imperfections can occur, such as breakup and eventual fracturing of the wave front. Such an effect has been demonstrated by numerical simulation of the above discrete subexcitable medium. The spatiotemporal behavior has been simulated for a coupling constant slightly below the critical coupling $K_{0}$. Initially, a strip of elements has been excited and a refractory layer has been added to avoid propagation of excitation to the left (see Fig. 3).

In Figs. 4, we show a series of snapshots of firing activity in the absence of noise as times elapses. These images show that the excitation wave initially propagates, but then shrinks and eventually disappears.

In Figs. 5, three time-lapse snapshots of the array are shown for the variance of the noise, $\sigma^{2}=0.08$. The wave now no longer shrinks, but evolves into a rotating spiral wave (only the core is shown). In contrast to the conven-
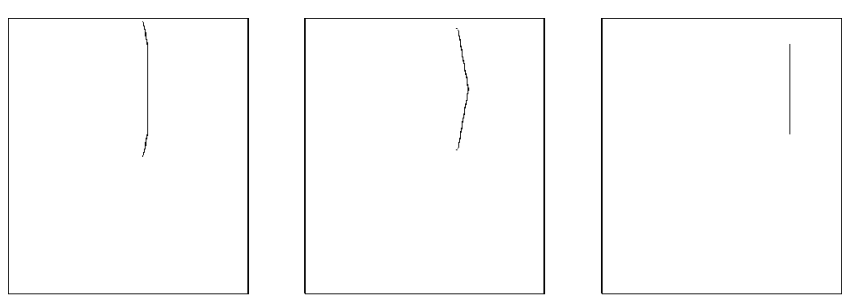

FIG. 4. The formation of a spiral wave in an array of $400 * 400$ threshold elements out of an initially excited column of elements for $K=0.151$, $\gamma=0.5$, and $\lambda=0.1$. A layer of refractory elements was attached to the left side of the initially excited column. The frames from the left to the right represent snapshots of firing patterns of threshold elements at positions $(i, j)$ with $i, j=1, \ldots, 400$ as time evolves. The dots denote firing elements. This sequence of snapshots has been obtained in the absence of noise. 

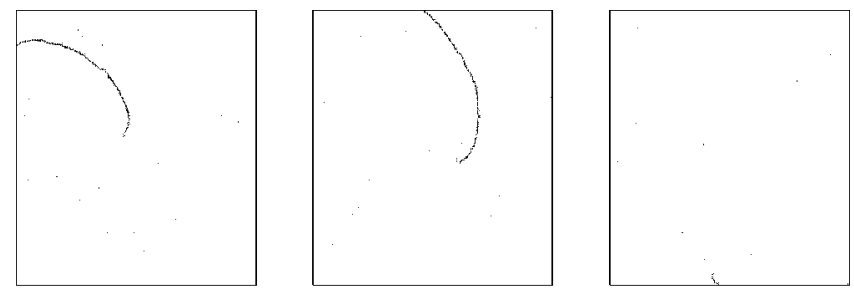

FIG. 5. The same as in Fig. 4, but with variance of the noise $\sigma^{2}=0.08$.

tional superthreshold spiral waves, the wave front is fuzzy and the core migrates randomly through the array.

In Fig. 6, the variance of the noise is increased to $\sigma^{2}$ $=0.13$. The evolving rotating spiral wave now has a larger curvature and more of the wave is now visible in an array of the same size. The breakup of wave fronts and fracture of the waves at high noise levels is demonstrated in Fig. 7 for $\sigma^{2}$ $=0.15$.

The snapshots in Figs. 4-7 demonstrate the ambiguous function of noise in subexcitable media. At low noise levels (in comparison to the excitation threshold), noise aids the propagation of wave fronts and the formation of rotating spiral waves. The noise also critically controls the features of the spiral waves (Ref. 12). At higher noise levels, the wave fragments develop turbulent-like behavior (spiral turbulance).

In a recent experiment, traveling subexcitable waves have been observed in a photosensitive BZ reaction. ${ }^{15}$ As described in Sec. II, the light intensity controls the chemical production of $\mathrm{Br}^{-}$, that controls the excitation threshold of the $\mathrm{BZ}$ reaction. The experiments were carried out with thin layers of silica gel in which ruthenium (II)-bipyridyl, a light sensitive catalyst for the BZ reaction, was immobilized. ${ }^{29}$ The gel was continually bathed with fresh catalyst-free BZ reagents in a Plexiglas reactor in order to maintain the system in an unvarying nonequilibrium state. Gels were exposed from below to spatially homogeneous light transmitted from a video projector through a $460 \mathrm{~nm}$ bandpass filter. The light intensity was adjusted to an appropriate reference level for maintaining the system slightly below the excitability threshold. The illumination field consisted of an array of square cells, with the intensity in each adjusted at equal time intervals to random values above or below the reference level. The random intensities were Gaussian distributed with the mean amplitude centered at the reference intensity.

The effects of noise on wave propagation are shown in Figs. 8, where superimposed snapshots depict the evolution
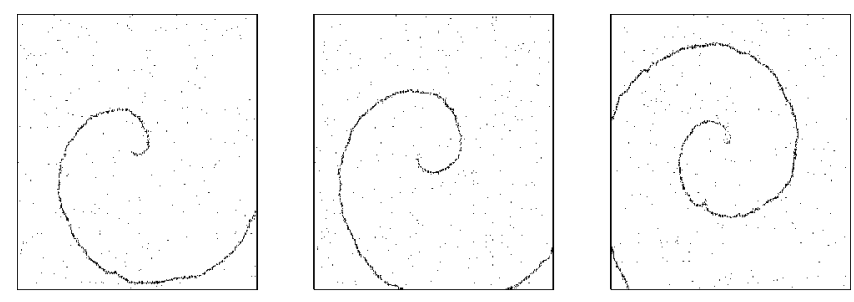

FIG. 6. The same as in Fig. 4, but with variance of the noise $\sigma^{2}=0.13$.
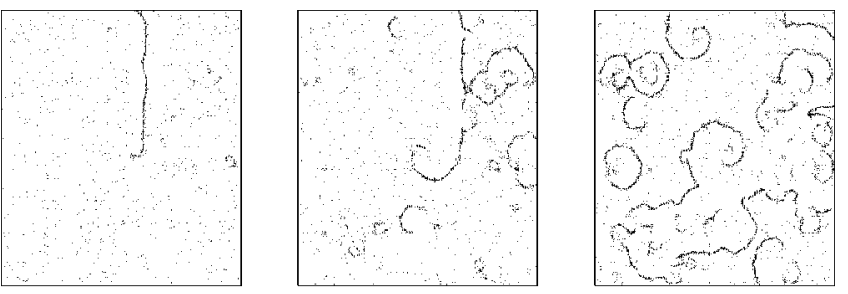

FIG. 7. The same as in Fig. 4, but with variance of the noise $\sigma^{2}=0.15$.

of a single wave segment at equal time intervals. In Fig. 8(a), we show superimposed snapshots of the wave in the absence of noise. The noise level (the standard deviation) is increased from panel (a) to (d). In (d) the noise is at the maximum level possible, which is determined by the reference intensity. A typical noise pattern is shown in Fig. 8(e).

The area of imposed noise in the illumination field consists of an array of square cells with the intensity $I$ in each adjusted at equal time intervals to random values according to $I=I_{0}\left[1+\left(\frac{1}{2}\right) G(x, \sigma)\right]$ with $|x|<2 \sigma$, where $I_{0}$ is the reference intensity and $G(x, \sigma)$ is a Gaussian distribution with a standard deviation of $\sigma$. The noise level, given by the value of $\sigma$, was varied between 0 and 1.0. The intensity was varied

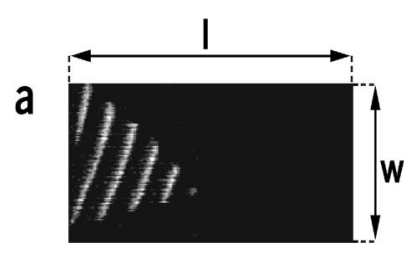

b

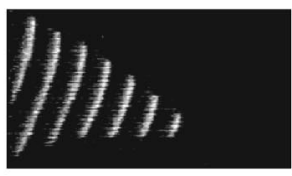

C
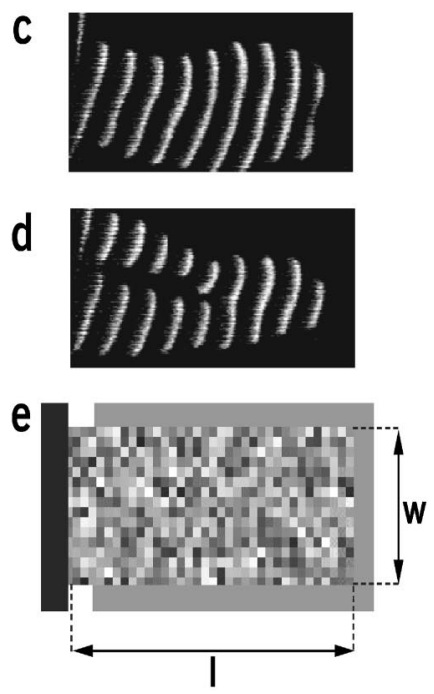

FIG. 8. Images of wave segments traveling through a BZ medium, perturbed by (a) 0 , (b) 0.3 , (c) 0.6 , and (d) 1.0 of the maximum noise level permitted by experimental conditions, and an example of the imposed noise array (e), with an adjacent excitable region (black) for initiating waves and the surrounding boundary (gray) at the subexcitable reference intensity. The superimposed snapshots show stroboscopically the behavior at $20.1 \mathrm{~s}$ intervals; the noise array was updated at $6.7 \mathrm{~s}$ intervals. 
on an eight-bit gray scale between 0 and 255. The cell size in these images was $4 \times 4$ pixels $(0.33 \mathrm{~mm} \times 0.33 \mathrm{~mm})$. Images were collected during a $50 \mathrm{~ms}$ interval between successive updatings of the imposed noise patterns. The composition of the $\mathrm{BZ}$ reaction was $0.27 \mathrm{M} \mathrm{NaBrO}_{3}, 0.05 \mathrm{M}$ malonic acid, $0.15 \mathrm{M}$ bromomalonic acid, and $0.15 \mathrm{M} \mathrm{H}_{2} \mathrm{SO}_{4}$. The silica gel medium $(0.3 \mathrm{~mm} \times 20 \mathrm{~mm} \times 30 \mathrm{~mm})$ was prepared by acidifying a solution of $10 \%(\mathrm{w} / \mathrm{w}) \mathrm{Na}_{2} \mathrm{SiO}_{3}$ and $0.5 \mathrm{mM}$ $R u(b p y)^{2+}$ with $\mathrm{H}_{2} \mathrm{SO}_{4}$.

Waves were generated in the adjacent region, with a lower light intensity (and higher excitability), at the left-hand side. In the absence of noise, waves entering the subexcitable region became shrinking wave segments [Fig. 8(a)]. Below the excitability threshold, free wave ends simply recede rather than form the rotating spiral waves typically observed in excitable media. ${ }^{12,13}$ Propagation failure occurs when the receding wave disappears, as shown in the final frame of the overlaid images. A slight enhancement of wave propagation is observed with low-level noise [Fig. 8(b)], in which the propagation failure occurs about one frame later. At higher noise levels [Fig. 8(c)], the wave segment no longer shrinks but propagates through the entire medium. At still higher levels of noise [Fig. 8(d)], fragmentation of the waves occurs.

The computer simulations of the discrete three-state model show qualitatively similar behavior. The reference states in both the experiment and the simulation are slightly below the excitability threshold. The three-state model at the value of the coupling used here generates noise-sustained spiral waves, while curling of the free ends of the waves is not observed in the experiments. Curling of the wave ends is also suppressed for a smaller coupling constant $K$ in the numerical simulations.

\section{THERMAL WAVES}

Both the experiments with the BZ reaction and the simulation of the three-state model described in the previous section were carried out at an operational point, where the systems were below, but very close to, the threshold for sustained wave propagation.

In this section, we consider the three-state model in situations when the coupling is substantially below the threshold for pattern formation, i.e., $K<K_{0}$. An initial preparation consisting of a strip of excited elements does not give rise to a large-scale wave sustained by the noise, but rather it fragments and disappears. After the initial excited strip has decayed, elements in the array fire in a random and unorganized fashion for a transient time. At later times, regardless of the initial conditions, a remarkable reorganization of the firing events sets in across the array, which manifests itself by the formation of clusters of firing elements. A sequence of snapshots is shown in Fig. 9 for $\gamma=1$ and $K=0.07<K_{0}$ $\approx 0.09$. In Fig. 9 (b), one can observe the spontaneous formation of a curved wave front, which resembles the core of a spiral wave. In the subsequent snapshots, we see the propagation of the front and its eventual disappearance [Fig. 9(d)]. These waves have been coined thermal waves, ${ }^{28}$ since they are sustained only by a thermal environment. They occur

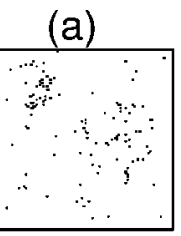

(c)

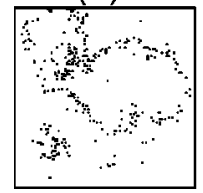

(b)

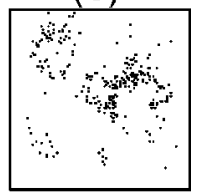

(d)

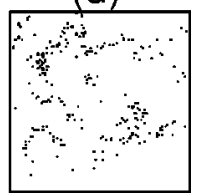

FIG. 9. Snapshots of evolving patterns are shown for $K=0.07, \sigma^{2}=0.15$, $\gamma=0.05$. The snapshots have been taken after a transient time of about 1000 time steps with 8 time steps between each consecutive snapshot. Time increases from (a) to (d). The array consists of $100 \times 100$ elements.

when the dissipation rate $\gamma$ is small, or, equivalently, when the local memory is long.

To quantitatively analyze the spatiotemporal properties of thermal waves, we introduce a novel method based on the analysis of coherent space-time clusters. ${ }^{28}$ In the first step, we stack a temporal sequence of $N_{t}$ snapshots of the medium, taken at times $t_{n}=n \Delta t$ to obtain a large space-time cube, which carries all the spatiotemporal information within the time interval $N_{t} \Delta t$. In the second step, we draw small cubes around each firing element with spatial side lengths $d_{s}$ and temporal side lengths $d_{t}$. Overlapping small cubes in the time-forward direction form objects that we have termed coherent space-time clusters. If two thermal waves collide to form one new wave, the coherent cluster corresponding to one of the incoming waves is terminated at the collision. The size $s$ of the coherent clusters, i.e., the number of firing elements whose small cubes build a cluster, is characterized by the cluster-size distribution function $p(s, t)$. The time dependence indicates that the distribution can change with time before a steady-state distribution is approached. The particular choice of the spatial and temporal side lengths $d_{s}$ and $d_{t}$, respectively, allows one to analyze the patterns on adjustable scales. Here, we have chosen the spatial side length $d_{s}$ such that the cubes of neighboring firing elements in space are overlapping, e.g., $d_{s}=1.3 a$. The temporal side length is chosen such that only the cubes of those firing elements overlap whose firing is likely to be causally related, e.g., $d_{t}=1$ (the normalized time step).

In Fig. 10, the cluster-size distributions are shown for $K=0.07, \quad \gamma=0.05$, and $\sigma^{2}=0.15$. For small times, $t$ $=10, \ldots, 60\left(N_{t}=50\right.$, filled squares $)$, the cluster-size distribution decays exponentially with increasing cluster sizes, indicating statistical independence of firing events. At later times (empty squares), regardless of the initial conditions, a remarkable reorganization takes place, which manifests itself by the formation of large clusters and a cluster-size distribution that scales like a power law, i.e., $p(s) \propto s^{-\alpha}$.

The exponent $\alpha$ of the cluster-size distribution is almost independent of the variance of the noise within a wide range. The observed variations are within the accuracy of the simulations. At a finite value of the dissipation rate $\gamma$, there is a critical noise strength $\sigma_{c}^{2}(\gamma)$, below which neither thermal 


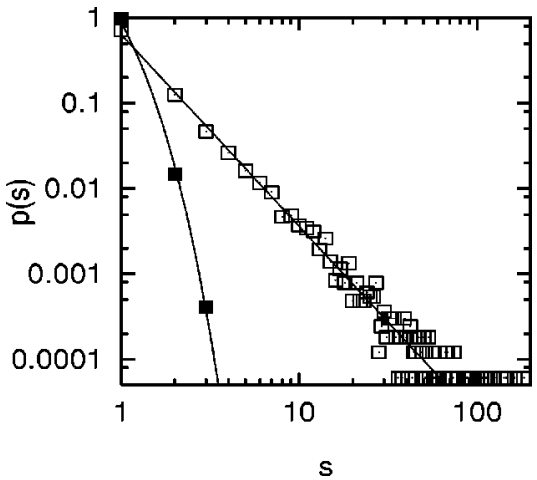

FIG. 10. Cluster size distributions are shown for $K=0.07, \sigma^{2}=0.15$, and $\gamma=0.05$ during the initial interval of the first 50 time steps (filled squares) and a later time interval (between 800 and 850 time steps), where the system has reached its steady state (empty squares). The lines through the symbols (actual simulation results) represent an exponential fit $p(s) \propto \exp \left(-s / s_{0}\right)$ for the initial-time interval and a power law fit $p(s) \propto s^{-\alpha}$ for the steady-state interval. The array used for the calculations consists of $200 \times 200$ elements.

patterns nor power-law scaling of the cluster sizes can be observed. Furthermore, the power-law scaling of the cluster size distribution is corrupted at large noise by an exponential cutoff, where the cutoff size is smaller than the system size. With a decreasing dissipation rate $(\gamma \rightarrow 0)$, however, the critical noise strength $\sigma_{c}^{2}(\gamma)$ approaches zero and the power law is limited only by the finite system size (see Fig. 11). For decreasing $\gamma$, the exponent $\alpha$ approaches $\alpha_{0}=2$ from above.

\section{THERMAL WAVES AND CALCIUM WAVES IN CULTURED NETWORKS OF HIPPOCAMPAL ASTROCYTES}

An expanded view of astrocyte physiology includes a role in the maintenance of neuronal microenvironments, but also implicates the astrocyte syncytium in long distance signaling based on propagating intercellular calcium waves. ${ }^{30-35}$ Calcium waves have been studied in hippocampal slice preparations and cultures of astrocytes. ${ }^{36-38}$

The main function of astrocytes in brain tissue is to absorb neurotransmitter (kainate) out of the synaptic cleft after

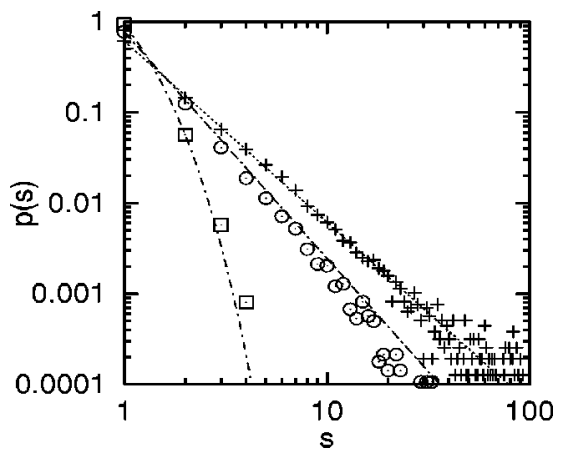

FIG. 11. Normalized cluster size distributions are shown for several dissipation rates $\gamma$ at constant variance of the noise $\sigma^{2}=0.2$. For $\gamma=0.005$ (crosses) and 0.05 (circles), we have fitted a power law through the points at small cluster sizes. The deviations from this line at large cluster sizes (cutoff) is visible at $\gamma=0.05$. The line through the points for $\gamma=0.1$ (squares) represents an exponential fit. The array used for the calculations consists of $200 \times 200$ elements. neural activity. As kainate binds to the astrocyte, $\mathrm{Na}^{+}$enters the cell. The $\mathrm{Na}^{+}$imbalance in the underlying cytoplasm builds over a period of 100-200 s, eventually triggering a reversal of the $\mathrm{Na}^{+} / \mathrm{Ca}^{2+}$ exchanger. Reversal of the exchanger favors exchange of extracellular $\mathrm{Ca}^{2+}$ for intracellular $\mathrm{Na}^{+}$. The influx of $\mathrm{Ca}^{2+}$, indicating the state of chemical imbalance, can be visualized using fluorescent dyes. The state of local imbalance can propagate wave-like along the surface of the astrocyte (calcium waves). Fluorescent imaging reveals space- and time-resolved information on propagating calcium waves.

In Ref. 39, cultured networks of hippocampal rat brain astrocytes (astrocyte syncytium) are exposed to various concentrations of kainate (to pretend neural activity). After an initial time interval, spontaneous formation of calcium waves can be observed under the microscope. The wave fronts are fuzzy and their shapes are fairly irregular. The waves start spontaneously at apparently random sites, indicating that their formation is similar to the noise-sustained thermal waves discussed in the previous section. Another feature similar to thermal waves is their spontaneous decay and their distribution of lifetimes. In the upper panel of Fig. 12, we show a sequence of time-lapse snapshots of the calcium activity of astrocyte syncitium with $10 \mathrm{mM}$ kainate. Subsequent images taken with a CCD camera have been subtracted from each other and coded in gray scale. High calcium activity is denoted by a darker gray. We observe mostly local calcium activity with very little spreading of the activity.

At $50 \mathrm{mM}$ kainate (see the lower panel of Fig. 12), we have selected a time-lapse sequence of frames depicting the core of a spiral wave with a significant spread before the wave disappears. In Fig. 13, we show a comparison of timelapse snapshots of the calcium activity and snapshots of simulations of the three-state model (active sites are denoted by black dots). ${ }^{40}$ Darker gray in the experimental figures indicates a higher calcium activity. The similarity is striking. In both sequences, we can observe the formation of a spiral core, its rotation and spread, and its eventual decay.

To apply the coherent cluster analysis to the calcium waves, we first have to apply a binary filter to identify calcium activity. The images furthermore are coarse grained to $100 \times 100$ cells, where each cell represents its average calcium activity. The binary filtered data have been analyzed ${ }^{39}$ with the coherent cluster method. The cluster-size distributions clearly show, much like the numerical data, a powerlaw decay (see Fig. 14). The exponent is-consistent with those of the models-slightly larger than 2 .

The agreement of the qualitative features of the calcium waves and the thermal waves observed in the simulations permits us to conclude that the calcium waves are actually sustained by the fluctuations in the cell network.

\section{DISCUSSION AND CONCLUSIONS}

We have demonstrated that noise can enhance spatiotemporal patterns in subexcitable media. This is a generalization of the notation of stochastic resonance to spatially extended systems. As SR in simple bistable systems requires a subthreshold signal, spatiotemporal stochastic resonance 


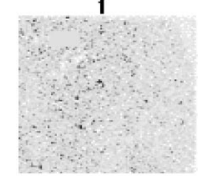

5
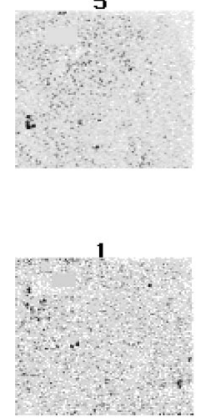

5

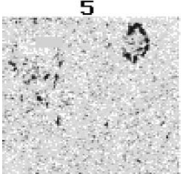

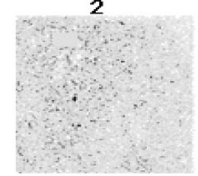

6
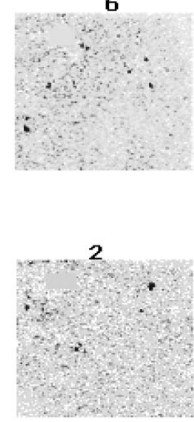

6

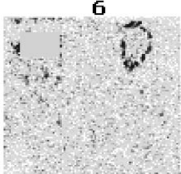

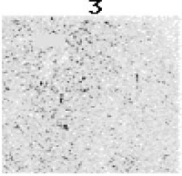

7
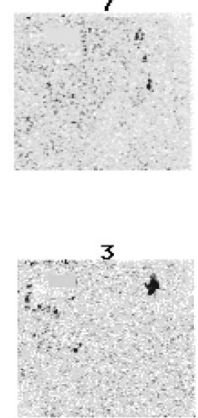

7

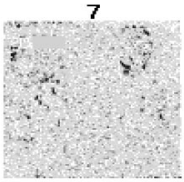

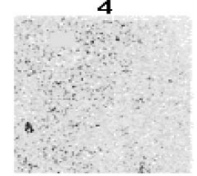

8
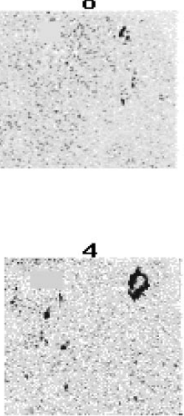

8

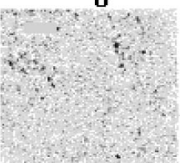

FIG. 12. The lower panel of difference images between consecutive frames shows a temporally evolving, rotating spiral wave. The wave is nucleated in the upper-left panel and dies in the noise in the lower-right panel. Time advances from upper left to lower right. The kainate dose was $50 \mu \mathrm{M}$. For smaller doses of kainate $(10 \mu \mathrm{m})$, we observe only small clusters of calcium activity.

(STSR) requires that the system is below the threshold of sustained pattern formation. An appropriate dose of noise allows the formation of patterns and the propagation of waves. Slightly below the threshold, noise can maintain large-scale patterns and determine their geometric features, $(a)$
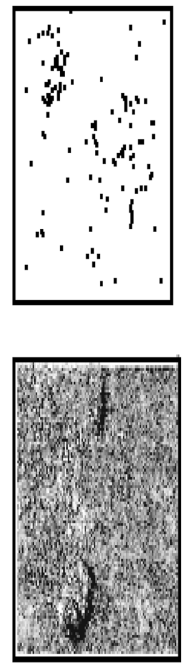

(b)
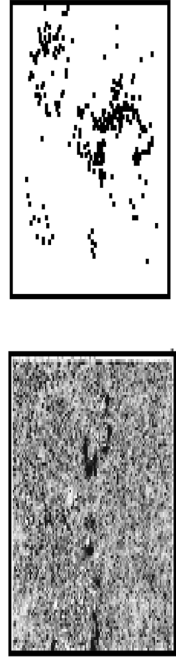

(c)
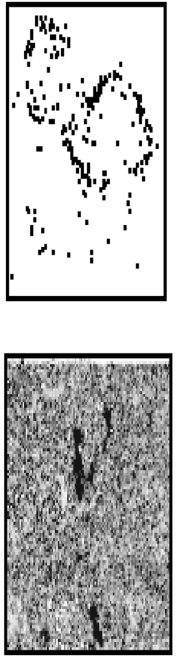

(d)
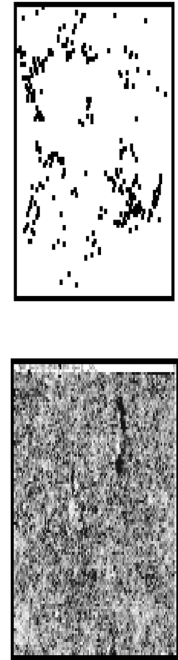

FIG. 13. Snapshots of evolving patterns produced by the numerical simulation (upper panel) are compared with snapshots of calcium activity. The system reached its statistical steady state during a transient period of about 1000 time steps of simulation. Snapshots were taken equidistantly with eight time steps between each consecutive pair. The array consisted of 100 $\times 100$ elements. Time increases from (a) to (d).

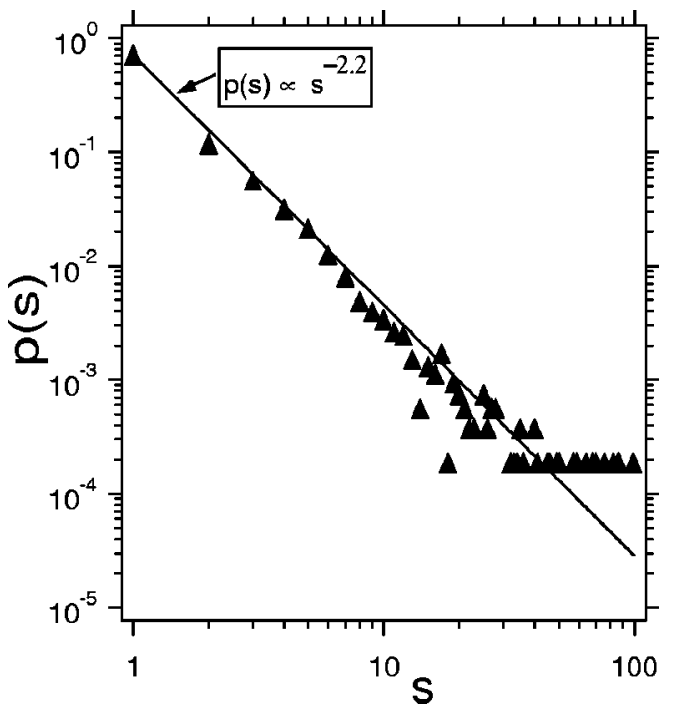

FIG. 14. The cluster-size distribution, $p(s)$, versus size, $s$, of the biological data (triangles) fit to a power law with an exponent $\alpha=2.2 \pm 0.2$.

as, for example, the curvature of spiral waves. Well below the threshold, noise maintains thermal waves, i.e., waves that appear spontaneously, spread for a statistical lifetime and then spontaneously disappear or collide with other waves. Locally, thermal waves show well-defined curved wave fronts, but on a larger scale they are turbulent.

Noise sustained waves have been observed in the photosensitive BZ reaction ${ }^{15}$ and in cultured networks of hippocampal astrocytes (calcium waves). The experiments with the photosensitive BZ reaction have demonstrated that noise can support the propagation of large-scale spatiotemporal patterns in subexcitable media, close to but below the threshold of excitation. A possible application of the subexcitable photosensitive $\mathrm{BZ}$ reaction is a novel design for detectors of moving objects when the image of the object is strongly obscured by environmental noise. Using some features of stochastic resonance in threshold-controlled systems, ${ }^{40}$ it has been demonstrated ${ }^{41}$ for a computer model of such a detector that the effective detection of images does not require more than a signal-over-noise ratio of about unity. Calcium waves in cultured networks of hippocampal astrocytes show many features (qualitative and quantitative) of thermal waves, i.e., noise maintained locally coherent but globally turbulent wave patterns. Especially interesting is the spatiotemporal organization of wave fragments reflected by the power-law distributions of the cluster sizes (including spatial extent and lifetime). Power-law distributed fragments indicate statistical self-similarity across the scales. The occurrence of larger clusters with a higher probability then just by chance (would yield an exponential distribution) indicates the possibility of statistically facilitated longe-range signaling between astrocytes. More concrete physiological meaning (in the context of brain pathologies) is currently investigated by the authors.

\section{ACKNOWLEDGMENTS}

This work was partially supported by the Deutsche Forschungsgemeinschaft (P.J.), the U.S. Office of Naval Re- 
search, Physics Division (F.M.), the National Science Foundation (K.S.), and the U.S. Office of Naval Research, Physics Division (K.S.).

${ }^{1}$ R. Benzi, A. Sutera, and A. Vulpiani, J. Phys. A 14, 1453 (1981).

${ }^{2}$ C. Nicolis, Tellus 34, 1 (1982).

${ }^{3}$ F. Moss, in Frontiers in Applied Mathematics, edited by G. Weiss (SIAM, Philadelphia, 1992).

${ }^{4}$ L. Gammaitoni, P. Hanggi, P. Jung, and F. Marchesoni, Rev. Mod. Phys. 70, 223 (1998).

${ }^{5}$ R. Benzi, A. Sutera, and A. Vulpiani, J. Phys. A 18, 2239 (1985).

${ }^{6}$ P. Jung, U. Behn, E. Pantazelou, and F. Moss, Phys. Rev. A 46, R1709 (1991).

${ }^{7}$ M. Inchiosa and A. Bulsara, Phys. Lett. A 200, 283 (1995).

${ }^{8}$ J. F. Lindner, B. K. Meadows, W. L. Ditto, M. E. Inchiosa, and A. Bulsara, Phys. Rev. Lett. 75, 3 (1995).

${ }^{9}$ F. Marchesoni, A. Bulsara, and L. Gammaitoni, Phys. Rev. Lett. 76, 2609 (1996).

${ }^{10}$ L. Schimansky-Geier and U. Sievert, in Stochastic Dynamics, edited by L. Schimaksy-Geier and Th. Pöschel (Springer-Verlag, Berlin, 1997), pp. 245.

${ }^{11}$ M. Löcher and E. Hunt, Phys. Rev. Lett. 77, 4698 (1996).

${ }^{12}$ P. Jung and G. Mayer-Kress, Chaos 5, 458 (1995).

${ }^{13}$ P. Jung and G. Mayer-Kress, Phys. Rev. Lett. 74, 2130 (1995).

${ }^{14}$ H. S. Wio, Phys. Rev. E 54, R3075 (1997); F. Castellpoggi and H. S. Wio, Europhys. Lett. 38, 91 (1997); J. M. G. Vilar and J. M. Rubi, Phys. Rev. Lett. 78, 2886 (1997).

${ }^{15}$ S. Kádár, J. Wang, and K. Showalter, Nature (London) 391, 770 (1998).

${ }^{16} \mathrm{M}$. Löcher and E. Hunt, submitted to Phys. Rev. Lett.

${ }^{17}$ R. Fitzhugh, Biophys. J. 1, 445 (1961); J. S. Nagumo, S. Arimoto, and S. Yoshizawa, Proc. IRE 50, 2061 (1962).

${ }^{18}$ J. J. Tyson and P. C. Fife, J. Chem. Phys. 73, 224 (1980).

${ }^{19}$ R. J. Field and R. M. Noyes, J. Chem. Phys. 60, 1877 (1974).
${ }^{20}$ H. J. Krug, L. Pohlmann, and L. Kuhnert, J. Phys. Chem. 91, 4285 (1989).

${ }^{21}$ T. Amemyia, S. Kádár, P. Kettunen, and K. Showalter, Phys. Rev. Lett. 77, 3244 (1996).

${ }^{22}$ P. Hänggi, P. Talkner, and M. Borkovec, Rev. Mod. Phys. 62, 251 (1990).

${ }^{23}$ A. Longtin, J. Stat. Phys. 70, 309 (1993).

${ }^{24}$ K. Wiesenfeld, D. Pierson, E. Pantazelou, C. Dames, and F. Moss, Phys. Rev. Lett. 72, 2125 (1994).

${ }^{25}$ J. J. Collins, C. C. Chow, and T. T. Imhoff, Phys. Rev. E 52, R3321 (1995).

${ }^{26}$ A. S. Mikhailov, Foundations of Synergetics I (Springer-Verlag, Berlin, 1994).

${ }^{27}$ J. D. Murray, Mathematical Biology (Springer-Verlag, Berlin, 1989).

${ }^{28}$ P. Jung, Phys. Rev. Lett. 78, 1723 (1997).

${ }^{29}$ T. Yamaguchi, L. Kuhnert, Z. Nagy-Ungvarai, and S. C. Müller, J. Phys. Chem. 95, 5831 (1991).

${ }^{30}$ A. H. Cornell-Bell, S. M. Finkbeiner, M. S. Cooper, and S. J. Smith, Science 247, 470 (1990).

${ }^{31}$ A. H. Cornell-Bell and S. M. Finkbeiner, Cell Calcium 12, 185 (1991).

${ }^{32}$ S. Boitano, E. R. Dirksen, and M. J. Sanderson, Science 258, 292 (1992).

${ }^{33}$ K. D. McCarthy and A. K. Salm, Neuroscience 41, 325 (1991).

${ }^{34}$ A. C. Charles, J. E. Merryl, E. R. Dirksen, and M. J. Sanderson, Neuron 6, 983 (1991).

${ }^{35}$ A. M. Jensen and S. Y. Chiu, J. Neurosci. 11, 1674 (1991).

${ }^{36}$ J. W. Dani, A. Chernjavsky, and S. J. Smith, Neuron 8, 429 (1992).

${ }^{37}$ A. C. Charles, E. R. Dirksen, J. E. Dirksen, and M. J. Sanderson, Glia 7, 134 (1993).

${ }^{38}$ S. J. Smith, Progress in Brain Research, edited by L. Hertz, M. D. Norenberg, E. Sykova, and S. Waxman (Elsevier, Amsterdam, 1992), Vol. 94, p. 119.

${ }^{39}$ P. Jung, A. H. Cornell-Bell, K. Madden, and F. Moss, J. Neurophys. 70, 1098 (1998).

${ }^{40}$ P. Jung, Phys. Lett. A 207, 93 (1995).

${ }^{41} \mathrm{G}$. Mayer-Kress (unpublished). 